Article

\title{
Facilitating Sustainable Waste Management Behaviors Within the Health Sector: A Case Study of the National Health Service (NHS) in Southwest England, UK
}

\author{
Jane Grose $^{1, *}$, Maria Bennallick ${ }^{1}$, Andrew Nichols ${ }^{1}$, Sabine Pahl ${ }^{2}$ and Janet Richardson ${ }^{1}$ \\ 1 Faculty of Health, Education and Society, University of Plymouth, 010, 8 Portland Villas, \\ Drake Circus, Plymouth PL4 8AA, UK; E-Mails: maria.bennallick@plymouth.ac.uk (M.B.); \\ andrew.nichols@plymouth.ac.uk (A.N.); janet.richardson@plymouth.ac.uk (J.R.) \\ 2 Department of Psychology, University of Plymouth, Drake Circus, Plymouth PL4 8AA, UK; \\ E-Mail: sabine.pahl@plymouth.ac.uk
}

* Author to whom correspondence should be addressed; E-Mail: jane.grose@plymouth.ac.uk; Tel.: +44-752-586-510.

Received: 6 March 2012; in revised form: 21 March 2012 / Accepted: 9 April 2012 /

Published: 12 April 2012

\begin{abstract}
Waste costs the National Health Service (NHS) £71.2 million in 2007/2008; recycling all papers, newspapers and cardboard produced by the NHS in England and Wales could save up to 42,000 tonnes of $\mathrm{CO}_{2}$. As the largest employer in the UK, the NHS is in a prime position to both lead the way towards a sustainable future, but also act as a test bed for organizational change and provide evidence of what works at an individual level to change attitudes and behavior. However these require changes in mindset, including values, attitudes, norms and behaviors which are required along with clear definitions of the problems faced in terms of economics, society and culture. Initial investigations of the literature indicate that behavior change theory may provide a feasible means of achieving constructive changes in clinical waste management; such approaches require further investigation. This paper describes a feasibility study designed to examine issues that might affect the introduction of a behavior change strategy and improve waste management in a healthcare setting. Guided by the evidence gained from our systematic review, 20 interviews were carried out with senior managers, clinicians and support staff involved in the management of healthcare waste from a broad range of agencies in South West England. Interviews were audio-recorded and transcribed for analysis. Thematic content analysis was conducted in order to identify key issues and actions. Data extraction, coding and analysis were cross checked independently by the four members of the research
\end{abstract}


team. Initial findings suggest tensions, between Government and local policies, between packaging and storage space at ward level and, and between the operational requirements of infection control and maintaining appropriate and ethical patient care. These tensions increase pressures on staff already trying to maintain high quality care in a resource restricted and changing environment.

Keywords: healthcare waste; infection control; management systems; procurement; behavior change

\section{Introduction}

Waste cost the National Health Service (NHS) $£ 71.2$ million in 2007/2008; recycling all paper, newspapers and cardboard produced by the NHS in England and Wales could save up to 42,000 tonnes of $\mathrm{CO}_{2}$ [1]. As the largest employer in the UK the NHS is in a prime position to both lead the way towards a sustainable future, but also act as a test bed for organizational change and provide evidence of what works at an individual level to change attitudes and behavior. These issues are supported by the NHS Sustainable Development Unit's (NHSSDU) recent report Sustainability in the NHS: Health Check 2012 [2] in which NHS leaders stated that "the areas they found more challenging included infrastructural changes, ensuring sustainable pathways and models of care, and working with other groups and organizations to achieve more sustainable results". Initial investigations of the literature indicate that behavior change theory aimed at both systems and individuals may enable the development of drivers which introduce a reduce, reuse and recycle philosophy to the management of healthcare waste $[3,4]$. As a first step there is a need to understand the experience of staff in healthcare, across the public and private divide, in terms of the obstacles to behavior change and the current efforts to remove those obstacles. This paper describes one stage of a four stage feasibility study which included a systematic review (complete), an interview study (reported here) an observation study (in progress) and will include an intervention. This second stage, the interview study, was designed to examine issues that might affect the introduction of a behavior change strategy to improve waste management in a healthcare setting.

The first stage of the feasibility study, the systematic review explored the extent of current research in relation to healthcare waste management. The review results indicated the need for:

- an understanding of the mind set of individuals,

- recognition that NHS employees who recycled at home were also more likely to do so at work,

- recognition that self-reports of behavior do not necessarily translate into actual behavior; and,

- behavior change theory is needed to drive system reorganization.

\section{Behavior Change}

Behavior change models have been developed from the social sciences, psychology and the health arenas. All three disciplines describe how individuals affect, and are affected by, their culture and environment. If "health" has been defined as "living in dynamic balance" [5] then the balance is 
maintained by the environment, in which people live and the behaviors they adopt, depending on cultural norms (behavior patterns that are typical of specific groups) [6]. To change behavior, cultural norms have to be understood both by the researched and the researcher. To maintain changes in behavior individuals first have to have the ability and willingness to change (choice) and second need to be supported to maintain that change (culture). More recent models of behavior change suggest that changes in the environment can cause behavior change directly [7]. There are two interlinking theories, first the behavior change perspective which focuses on individual behavior and second system based theories of change which act as guides when planning system wide interventions.

For behavior change to be sustained managers need to understand how their organization's policies and culture affect individual behavior and establish routines and environments in which the "right" behavior is easily carried out and habits are likely to be established. To achieve this, a system-wide approach to considering specific elements in a change process needs to take place. As a first step there is a need to understand the reasons for existing behaviors and provide evidence for an intervention to address possible change.

\section{Methods}

Guided by the evidence gained from our systematic review, the second stage of the feasibility study involved twenty in depth qualitative interviews with senior managers, clinicians and support staff involved in the management of healthcare waste from a broad range of public and private, health advisor and health provider agencies in South West England. The research took place in between April and June 2011. The study settings included a private hospital, an NHS (Government funded) hospital and a number of care homes (offering residential and day care for the elderly). The reason for choosing a range of organizations from within and outside the NHS, and across the public and private divide, was that we were interested in understanding how current waste management practices were managed and to gather evidence of the limitations and successes in a variety of arena. It was hoped this would provide breadth and depth to the evidence. We initially contacted heads of department in the organizations we wished to be involved in the study. These organizations were selected following consultation within the research team and with colleagues working on sustainability in the University. The heads of department identified senior staff involved in the management of waste. Participants were therefore purposively sampled to include a range of views from each of the organizations and different types of staff $(n=20)$ [8]. The participants' occupations included public health clinicians with a responsibility for coordinating waste; local Government officials; operational managers in public and private healthcare providers, senior and junior nurses and portering staff.

The semi-structured interviews, using a structured interview guide, took place in venues and at times negotiated with, and convenient for, the participants. Interviews ranged from between $45 \mathrm{~min}$ and $1 \mathrm{~h}$ duration. Participants were reminded that all data they provided were coded to protect their identity and to allow them to speak candidly [9]. The structured interview guide included demographic information; background/context/information, culture of the organization/barriers/possibilities for change; specific reduce, re-use, recycle questions and the participants attitudes towards such issues as climate change and sustainability. 
All the interviews were digitally recorded and transcribed. Thematic analysis [10] was conducted in order to inductively develop codes and themes. Data extraction, coding and analysis were cross checked independently by members of the research team. The researcher read and re-read the transcripts identifying areas of concordance and divergence across the interviews. This enabled both the breadth and depth of the data to be thoroughly investigated and all interview data to be part of the analysis. Overall themes were developed following discussion of the initial findings with members of the research team. Study rigor was established through the use of a decision trail following the principles of credibility, transferability and dependability [11]. An interim report was sent to all participants for comment and these were incorporated into the final report.

\section{Ethical Approval}

All processes were informed, designed and executed in full accordance with established principles for research involving human participants. Guiding principles for designing and carrying out research were adhered to, these include respect for all individuals involved in the research, valid consent, openness, honesty, right to withdraw, and confidentiality (Nursing and Midwifery Council's Code of Professional Conduct 2008). There was no involvement of patients in this research, but as it involved NHS staff within healthcare environments, the approval and guidance of the local NHS Research Ethics Committee was sought and received.

\section{Findings}

The findings identified a complex pathway of confounding factors which led to the vast quantities of waste being produced by organizations involved in the study. It was not simply an issue of individual members of staff putting non-infectious items in clinical waste bags which appeared to cause an unnecessary increase in the amount of healthcare waste being removed. The analysis of the data from twenty in-depth interviews across a range of organizations developed three themes: (1) systems; (2) attitudes and behavior; and (3) the way forward. This paper reports the first of those themes because the evidence from the interviews described the pressures on staff from outside their organizations and how these pressures were manifested within the organization, preventing them from responding to guidance, and reducing the possibility of positive behavior change. This theme is seen as worthy of reporting separately from the other two themes, which will be reported on elsewhere, because, as has been said, behavior change relies on the culture and environment in which people work enabling them to adapt practice. This theme describes the difficulties they face because of external conflicting advice and guidance, how and from where items are purchased and packaged and the nature of current health provider organizations.

\subsection{Key to Quotations from the Transcripts}

Number of transcript $=$ first number in code

Line in transcripts $=$ second number in code

[ ] $\quad=$ some text has been removed to maintain sense of the chosen quote

$\mathrm{xxx}=$ some text has been removed to maintain confidentiality of names and places 
In the first part of the interviews we felt it was important to ask participants to define what they understood by the terms reduce, re-use and recycle and these terms were generally understood. Some described it as just "good housekeeping" and many pointed out that this process was not new "make do and mend" had been commonly understood for many years.

Recycle would be to taking a material and effectively doing some sort of recovery or re-processing of it to use that material for something else. So you could take a used aluminum can that can be mangled up and re-smelted and made into new aluminum products, [ ]. So that would be recycling. Reuse would be using it again for its original purpose. So you're taking a can and you're cleaning it out and you're using it as a can again. Reduce would be, [ ] in essence you're trying to reduce what waste you produce in the first place. So it might be you're using less in your process or your process is more efficient as such. [ ] Say you have a simple process, sheets of cardboard are being fed through and you're punching out shapes of cardboard, simply by arranging your punches better or getting the right size cardboard you reduce the amount of waste cardboard you generate by most efficient placement, best usage of material. 5:300

With this basic understanding the realities of developing a culture based on the three R's led to the following evidence base which identified issues which would need to be dealt with before culture change was a possibility.

\subsection{Legislation and Guidance}

The data provided a description of how individual organizations managed their waste collections and how in some cases private and public organizations were working together in some small ways. The majority of organizations had issues with space management (even in office based rather than health care institutions). They also described difficulties in providing clear up-to-date information which was relevant and delivered in a form that was appropriate to the needs of different groups. In addition there were general issues with the location of bins and the removal of waste. However the sense of urgency to get to grips with the problem was apparent across the data:

... organizations know they've got to take this agenda seriously. But there are still elements, I think there are still, we know we've got to take this agenda seriously but we're still working in the old ways. 18:196

Interviewees were concerned with the vagueness of what constituted clinical waste. There appeared to be tensions between infection control guidance and waste management guidance, the former concerned with protecting patients and the latter with health and safety at work:

I think having a clear definition of what clinical waste is would help. ... We got the regulations that were broken down into hazardous, infective, non-infective, clinical-all these different terminologies. And that's just recently been revised. It would say things like, bodily fluids from a non-infective patient, but how do you know somebody is non-infective, because as infection control practitioners we would always advise that all bodily fluids be treated as potentially infectious by nature. 15:95 
The problems with definition were compounded by inconsistent approaches:

I mean one of the fundamental weaknesses here is that we haven't had a consistent or coordinated approach to waste for the organization which, considering the size of our organization, is a bit of a travesty, really. 4:288

It was suggested that these two interest groups (infection control and waste departments) tended to work in silos meaning that staff received conflicting advice. It was apparent from the interviewees in advice provider organizations that "good NHS Hospitals" were those who had invested in environment/waste committees which included a range of staff and provided on-site immediate advice on how to manage specific items of waste and were thus able to respond quickly when staff needed help to make changes at department and unit level. It was suggested that hospitals that had made those investments made sufficient savings in reduction in waste produced to make them financially viable:

The good Trusts (Hospitals) are very good but there is a gap, there are those Trusts that monitor legislation and appliance across everything, not just environment, and you'll see them moving with the times. And there are those that are probably still back in the days of Crown immunity and what we're seeing is the gap between the good ones and the bad ones opening up. The good ones continue to progress with the changes in legislation, the increasing dependence or drive for sustainability. So their procedures are continually improving, they're doing more and more and they're building on their past successes. 5.533

Organizations that were not dealing with environmental issues so well, tended to have poor leadership on the issue and lack of training and support.

If I was able to do one thing now it would be to pick up the competence of the waste managers in the NHS. Because although there are some very good ones, and there's quite a reasonable numbers of them, there are a lot who are sat out there on their own without senior management support, with limited training and expertise, very few places to go for help and little money in their departmental budgets. And training for them and free tools for them to use within their Trust so they're not hitting the budgets, would, could make a huge difference to performance out there. 5: 691

\subsection{Procurement and Manufacturing}

Many of the interviewees raised the problem of the amount of packaging that equipment was wrapped in when it arrived at ward level. Managing cardboard and other packaging materials seemed to be a major issue amongst all the organizations involved in the study. Some organizations had reduced the amount of products arriving on site by instigating a strict ordering system, only ordering what was necessary on an individual patient basis and managing a detailed stock control:

Some (residential) homes allow the pharmacists, the chemists, to re-order everything, and they will just tick every box and re-order, because obviously they're going to gain by that I would imagine. [ ], because we actually control our stock. [ ] We have xxx quality support I think they're called, and they come and do an audit every so many months in every home, and they check if we're holding too much stock. [ ] l we're not buying them in. Again we're saving the 
NHS money, which again is our money. It's good practice anyway to do that and not to have too much stock. It's actually criminal, the amount of waste through drugs. It really is quite horrendous. I have, as a community nurse in my previous job, gone into homes, big homes, and they've had bags and bags and bags of dressings, and again they're not allowed to use them for anybody else. They just have to be thrown. It's shocking really. 20:410

We asked interviewees about the types of waste they handled and the methods they used to dispose of it. The types of waste created depended on the environment in which people were working. Many of the interviewees were office bound so they needed to manage, paper (both confidential and general), cardboard, plastics, foil and food waste. Health service providers, in addition to general waste, had to manage latex, polythene, linen, sharps, all of which might carry infectious material and then human tissue from operating theatres. Each item used came wrapped in a variety of packaging materials:

Procedure packs without their sharps, of course, so dressings packs etc. Lots and lots of it is just boxes, packets, syringe packets from opening syringes. Because all of our syringes are single use and come singly wrapped. Lots of polythene, plastic, cardboard, loads and loads of stuff that you wouldn't classify as clinical waste normally. Huge boxes that equipment comes in. Lots of paper, masses and masses of paper, which we do separate mostly, and that goes as confidential waste. $14: 144$

Of note was that in the NHS staff felt the packaging issue was not in their control, that they were not able to influence policy to a level where manufacturers would be taken to task for unnecessary packaging:

I think it's out of our hands. If you had a word with one of the store people who obviously do the ordering and oversee the arrival of various items that are used in theatre and on ward, it's the way it's packed. It might come from abroad, and every box for a little piece of equipment that theatre might use, it's got a how to use manual. Every box has got one of those. So you're throwing the waste paper away. It's obviously never used, never looked at. 16:286

Yet there was a general feeling that in private healthcare organizations or small businesses there might be a possibility to negotiate with manufacturers or, if that had no effect, refusing to buy items with too much packaging:

If you are in an organization, in a large organization, sat there doing a task whether it's in a factory, in a hospital or whatever, that's producing a waste, your ability to influence reduction through procurement is almost nil. In a small organization, if you're a small business man, the cost of that affects you directly and you can directly influence procurement. You say, I'm gonna buy smaller packaging so I waste less, so it doesn't cost me any more. And you can go and do it. The bigger the organization, the harder that is to feed through unless you've got very, very good systems. 6:597

\subsection{The Health Provider Organization}

Apart from the difficulties in managing excess cardboard and other packaging materials, the interviewees identified areas of concerns about the buildings in which they worked and the way 
systems had been set up to manage waste. In the first instance staff were working in buildings which were no longer fit for purpose. New builds offered the possibility of incorporating a range of adaptations which might improve the storage and management of waste. In existing buildings as patient/client throughput increased, the resulting increase in activity increased the waste produced. This increased throughput creating challenging problems relating to how they disposed of waste and where they could site bins to make sure, at a minimum, rubbish was put in a bag even if it wasn't the correct color bag.

In both old and new buildings interviewees identified the lack of space for recycling or storage of waste. Lack of space meant that choices needed to be made about placement of bins. For example if there was only space for one bin, in order to protect patients, the one bin was designated clinical waste and all waste was put in that bin whether or not it was infected. This then had to be dealt with as clinical waste and therefore incurred unnecessary costs.

It's very hard to put systems in place in a lot of areas because we don't have the room. If you go into say an anesthetics room, which we would love to do some recycling in, there is no room to put any other bins. There's not room for what there is at the moment. Unfortunately a lot of our buildings are elderly. This one was built in the '60s, in '65 I think, so it was designed for a different time. And now they could do with more room, more storage, and that is an issue almost across the board, storage, you know, room and space to keep things. That is one of the biggest barriers to recycling and having correct bins in place. 8:377

Even where there were bins, where they should be located remained an issue. If clinical waste bins were placed near to hand wash basins then paper towels would inevitably be put in them. With limited space and the need to maintain a clean environment for vulnerable patients sometimes choices had to be made between infection control and the reduction of clinical waste:

Placement is one thing, placement of bins. You wouldn't want a clinical waste bin next to a sink unless it was a special area which was dealing with people with a highly contagious disease. So placement is one thing and education is another. 6:628

This issue of poor separation was not just related to space and location of bins, when we questioned those who were responsible for auditing the separation of waste they felt that the definition of clinical waste had changed, or was unclear, so this affected people's understanding of what to do with it:

I think clinical waste over the years, or the understanding of the concept of clinical waste has probably changed quite a lot. Because in the past you would put gloves and aprons in the yellow bags regardless. You'd put paper hand towels in the yellow bags, regardless. And again, in a previous working life we had yellow bags everywhere on the wards. We didn't have any mixture. It was all yellow bags. The thought process behind that being, 'It's been on a ward. It's got to be clinical waste'. 15:434

Yet when we discussed this with staff providing direct care in vulnerable areas they had no choice but to put aprons and gloves in clinical waste bag, because there was no space for a range of different colored options: 
We call things clinical waste that aren't and we do that for instance in our bathrooms because we have a lot of ladies who've just given birth and so we could get a lot of blood stains, domestic waste. So therefore we've identified our bathrooms, well our two bathrooms, as clinical waste, even though most of what goes into that bag is not clinical waste at all. And you could say we put two bins in there but the toilet here is like three foot square and there's no room for the bin it's got, let alone a second one. 14:120

Service reconfiguration and waste disposal behavior was therefore hampered by the age of the building and the management of space, storage and packaging. When these issues were raised participants raised concerns about finding resources to make changes at a time of financial constraint.

\subsubsection{Costs}

Inevitably with all budgets in the NHS being reviewed and the need to relate activity to costs in private or public health care providers the issue of how to keep costs down was a priority for managers. Private providers had more accurate information about activity costs in relation to waste than NHS care providers. Some NHS providers, although concerned about reducing the amount they sent to landfill, were not aware of alternatives. It was pointed out that as the cost of landfill would keep increasing this may eventually force Trusts to recycle more. National pressures on price in relation to depleted oil reserves may lead to oil based products becoming too expensive [12]. The knock on cost of buying these expensive items would mean that Trusts would have to find alternatives or further prioritize the kind of care they can afford to provide.

...unless we start to get a handle on the sensitivity to things like the oil price ...we're going to suddenly find ourselves in the situation where last year we did ten operations, this year we can only afford to do eight operations because the cost of products that we're using in that arena have gone up so much. 18:208

\section{Discussion and Conclusions}

Deciding how to manage the disposal of healthcare waste in a system which is functioning to capacity presents a range of issues. Over-packaging and lack of space, the location of bins and the high turnover of patients create pressures on the health care system and on individual staff members. In this study staff were fully aware of the need to reduce packaging, re-use items where possible, and send waste for recycling, they did so at home, but at work competing pressures meant that waste disposal was not a priority. The results from the systematic review found that people who recycle at home were more likely to recycle at work [3], however our interviewees suggested this was only possible when it was easy to do so. This study has highlighted some of the difficulties which prevent them from separating waste at ward or unit level. The advice provided to the UK NHS on recycling of waste [13] needs to take into account the pressures at ward level and provide more innovative methods of separation which require little effort or space but enable staff to manage waste effectively.

With system issues affecting behavior some incentive might be required to encourage people to change their recycling behavior. Incentives and disincentives are used in other areas where individual behavior change is necessary (such as smoking and obesity) yet there are concerns about their 
effectiveness [14]. On the one hand an incentive can be seen as rewarding bad behavior and on the other a disincentive can have the effect of alienating the group it is intended to reach. In healthcare goal setting and rewards have been shown to influence behavior at department or ward level [15] yet these changes can be short lived after the reward has been provided. Involving staff in appropriate rewards for changes in systems or practice can be beneficial. Of greater sustained value appears to be the system wide approach to gaining International Organization for Standardization (ISO) awards which can be audited over time. It should be recognized though these are expensive to achieve and may alienate staff if the changes necessary to receive the award entail an increased workload.

The Audit Commission report [16] which provides an account of the UK NHS response to sustainable procurement comments "Many processes known to reduce procurement costs such as consolidating orders and invoices, rationalizing the supplier base and reducing the number of different makes of products are still not being taken up by many Trusts". This interview study data confirms the findings of the Audit Commission report and further describes the particular issues which staff have to manage at ward level. Ordering needs further rationalization and manufacturers need to be challenged to explain why individual items are packaged to such a degree that the amount of waste produced far exceeds the size of the item being used.

The range of issues raised by this interview study is complex and calls for leadership commitment but also the need to hear staff on the frontline who are working in often very challenging situations. Space, lack of coordination between infection control and waste management and time pressures prevent them from fully adopting a change in practice. We have identified manufacturing and procurement as a starting point for relieving the pressures at ward level. Reducing packaging would free storage space and create more space for patient care. Diagram 1 describes this pathway.

Diagram 1. A sustainable procurement pathway.

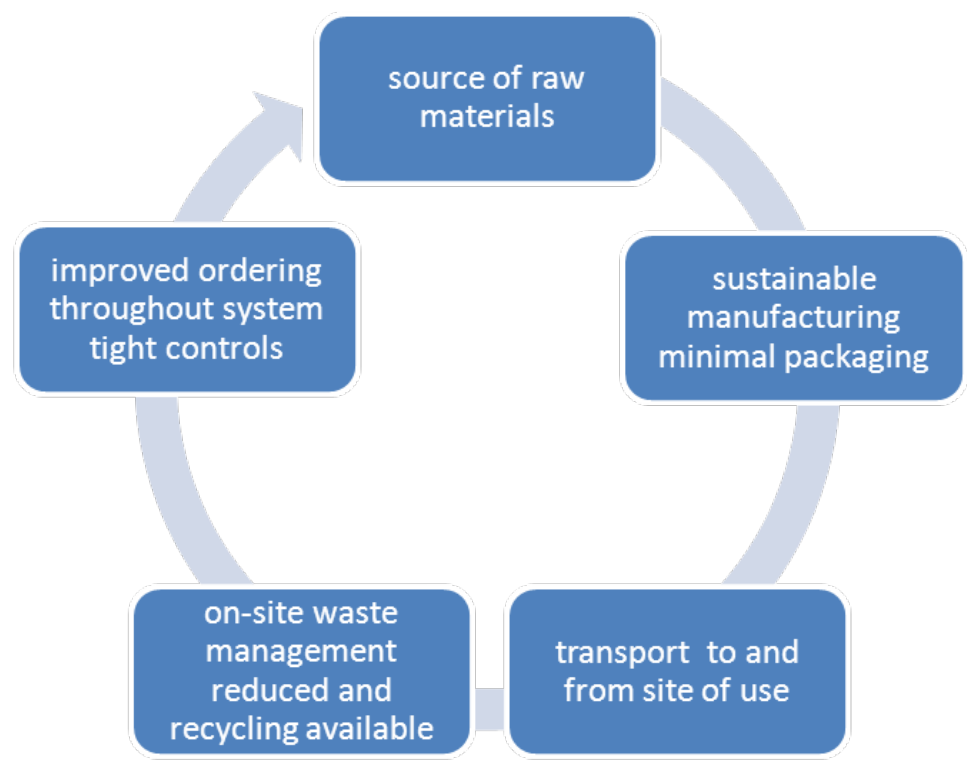

The pathway tracks the stages involved in reduction of packaging: (1) the source of raw materials for manufacture should be focused on sustainable products, only using what is necessary for the product; (2) minimal packaging of items would allow economies of scale; (3) transport throughout production and delivery focused on minimizing trips, and sourcing products closer to home; (4) on-site 
waste management will reduce if there is a reduction in packaging; (5) tightly controlled ordering and procurement systems will drive down costs as customers insist on products which have minimal packaging. The whole system should be constantly monitored and evaluated so that minimum levels of procurement and packaging are maintained.

There is a need for a flow of information from staff to legislators which will enable constant evaluation of the systems in place to manage waste. We also found that health care provider organizations both public and private would benefit from local collaboration. For example we found private healthcare providers had greater knowledge of activity-based costing than their public health care provider colleagues and public health care providers had equipment such, as cardboard compactors, which could be shared across public and private sites.

Government and non-governmental organizations have developed a range of guidelines and policies to both tackle climate change and create a more sustainable health service $[17,18]$. There are many organizations working to audit and monitor how this legislation is put into place and financial savings because of these changes have been significant. What this study adds, to existing evidence, is an in-depth understanding of staff responses to multiple directives and the problems and difficulties created by the systems that are designed to help dispose of healthcare waste effectively and efficiently. The relevance of these findings is that talking about increasing $\mathrm{CO}_{2}$ emissions or creating new guidelines is not necessarily the way to influence behavior change. Finding what is meaningful to individuals, for example dealing with packaging at ward level or the management of space for patients and waste bins, will have a greater impact on how people behave because it improves the environment in which they work. All the participants were aware of how things could improve so the first stage in a behavior change approach is in place. What is needed now is to work with manufacturers, to re-configure space to allow a range of bins to be provided, and to listen to staff about what is and what is not possible.

\section{Limitations of This Research}

This research was undertaken with a small sample in organizations in South West England and so the findings are not generalizable. However the findings do offer areas for future investigation. During the research it became clear that sharing the evidence with local manufactures would be a valuable next step in the research program to discuss ways of reducing packaging and developing items which can be re-used. The research team is currently engaged with one local manufacturer to that end.

\section{Recommendations}

1. The advice provided to the UK NHS on recycling of waste needs to be reviewed to take into account the pressures at ward level;

2. Working with frontline staff, and waste container manufacturers, more innovative methods of separation need to be developed which require little effort or space but enable staff to manage waste effectively;

3. Ordering needs further rationalization and manufacturers need to be involved in future research to explore why individual items are packaged to such a degree that the amount of waste produced far exceeds the size of the item being used; 
4. The source of raw materials for manufacture should be focused on sustainable products, only using what is necessary for the product;

5. Transport throughout production and delivery should be focused on minimizing trips, and sourcing products closer to home;

6. Better networking and collaboration between infection control and waste management, at both policy and practice levels, would enable waste to be managed and patients to be protected;

7. There is a need for a flow of information from staff to legislators which will enable constant evaluation of the systems in place to manage waste;

8. In order to achieve sharing of information there needs to be local collaboration across organizations based on enabling the three R's to become imbedded into organization's practice;

9. Legislators and healthcare waste managers need to listen to frontline staff (especially nursing and portering staff) about what is and what is not possible.

\section{Acknowledgments}

We would like to thank the representatives of the organizations who took part in this interview study and we are grateful for their continued support and advice as the program of research progresses.

\section{Conflict of Interest}

The authors declare no conflict of interest.

\section{References}

1. Marmot, M. Fair Society, Healthy Lives: Strategic Review of Health Inequalities in England Post 2010; Department of Health: London, UK, 2010. Available online: http://www.marmotreview.org (accessed on 10 August 2011).

2. NHS Sustainable Development Unit. Sustainability in the NHS: Healthcheck 2012; Department of Health: London, UK, 2012. Available online: http://www.sdu.nhs.uk/downloaded (accessed on 20 January 2012).

3. Tudor, T.L.; Barr, S.W.; Gilg, A.W. A tale of two locational settings: Is there a link between pro-environmental behavior at work and at home? Local Environ. 2007, 12, 409-422.

4. Tudor, T.L.; Barr, S.W.; Gilg, A.W. A novel conceptual framework for examining environmental behavior in large organizations: A case study of the Cornwall National Health Service (NHS) in the United Kingdom. Environ. Behav. 2008, 403, 426-450.

5. Abelin, T.; Brzezinski, Z.; Carstairs, V. Measurement in Health Promotion and Protection; World Health Organization Regional Office for Europe: Copenhagen, Denmark, 1989.

6. Terry, D.; Hogg, A.; McKimmie, B. Attitude-behaviour relations: The role of in-group norms and mode of behavioural decision making. Br. J. Soc. Psychol. 2000, 39, 337-361.

7. Bell, P.; Greene, T.; Fisher, L.; Baum, A. Environmental Psychology; Harcourt College Publishers: Orlando, FL, USA, 2001.

8. Marshall, M.N. Sampling in qualitative research. Fam. Pract. 1996, 13, 522-525.

9. Miles, B.; Huberman, A. Qualitative Data Analysis; Sage Publications: London, UK, 1989. 
10. Creswell, J. Qualitative Inquiry and Research Design; Sage publications: London, UK, 1998.

11. Guba, E.; Lincoln, Y. Fourth Generation Evaluation; Sage Publications: Newbury Park, CA, USA, 1989.

12. McCartney, G.; Hanlon, P.; Romanes, F. Climate change and rising energy costs will change everything: A new mindset and action plan for 21st Century public health. Public Health 2008, $122,658-663$.

13. Healthcare Waste Management: A Compendium of Good Practice; Report Department of Health, Stationary Office: London, UK, 2000.

14. Marteau, T. Judging nudging can nudging improve population health? BMJ 2011, 342, 263-265.

15. Swann, C.; Owen, L.; Carmona, C.; Kelly, M.P.; Wohlgemuth, C.; Huntley, J. A nudge in the right direction: Developing guidance on changing behaviour. In Evidence-Based Public Health: Effectiveness and Efficiency; Killoran, A., Kelly, M.P., Eds.; Oxford Press: Oxford, UK, 2009.

16. Procurement and Supply; Report; National Audit Office: London, UK, 2002. Available online: http://www.audit-commission.gov.uk (accessed on 11 October 2011).

17. Blenkharn, J. Standards of clinical waste management in UK hospitals. J. Hosp. Infect. 2006, 62, 300-303.

18 Strategy Unit. Waste Not, Want Not: A Strategy for Tackling the Waste Problem in England; Strategy Unit Cabinet Office: London, UK, 2002.

(C) 2012 by the authors; licensee MDPI, Basel, Switzerland. This article is an open access article distributed under the terms and conditions of the Creative Commons Attribution license (http://creativecommons.org/licenses/by/3.0/). 